\title{
AVALIAÇÃO DE IMPACTO AMBIENTAL NO CONTEXTO DA CONSERVAÇÃO DA BIODIVERSIDADE
}

\author{
ENVIRONMENTAL IMPACT ASSESSMENT IN THE CONTEXT OF BIODIVERSITY CONSERVATION
}

\begin{abstract}
Julieta Bramorski ${ }^{1}$
1 Professora Titular do Departamento de Meio Ambiente e Desenvolvimento - DMAD vinculada ao curso de bacharelado em Ciências Ambientais da Universidade Federal do Amapá. E-mail: bramorski@gmail.com Orcid: https://orcid.org/0000-0002-2783-5644
\end{abstract}

RESUMO: A Avaliação de Impacto Ambiental (AIA) possui papel central no contexto do licenciamento ambiental. Constata-se que valores da biodiversidade são frequentemente subestimados nos processos de licenciamento de empreendimentos de significativo impacto ambiental realizados no Brasil. Para abarcar minimamente questões relevantes em termos de biodiversidade, a Associação Internacional para Avaliação de Impacto recomenda que a AIA contemple diferentes níveis de análise para avaliar a viabilidade ambiental de atividades/empreendimentos. Para que esta cumpra seus objetivos é fundamental a inserção da componente biodiversidade no escopo dos estudos, em escalas espaciais e temporais adequadas, com grau de detalhamento coerente com questões a serem compreendidas.

Palavras-chave: Viabilidade ambiental. Processos de licenciamento ambiental. Biodiversidade. Impactos ambientais/Estudo de impacto ambiental.

ABSTRACT: Environmental Impact Assessment (EIA) plays a central role in the context of environmental
licensing. It is observed that biodiversity values are often underestimated in the licensing processes of pro-
jects of significant environmental impact in Brazil. The economic values of biodiversity, social values and
other intrinsic values are not usually considered. To address biodiversity-relevant issues minimally, the Inter-
national Association for Impact Assessment recommends that the EIA address different levels of analysis
to assess the environmental viability of an activity/undertaking. In order for the EIA to fulfill its objectives,
it is essential to include the biodiversity component in the scope of studies, at appropriate spatial and tem-
poral scales and in a degree of detail consistent with the issues to be understood. Keywords: Environmental viability. Environmental licensing procedures. Biodiversity. Environmental impacts. Environmental studies.

Sumário: Introdução - 1 Breve histórico da AIA no Brasil - 2 A AIA nos processos de licenciamento ambiental - 3 AIA no contexto da biodiversidade - 4 Desafios atuais e futuros - Considerações Finais - Referências

\section{INTRODUÇÃO}

A partir dos conflitos observados no período pós-guerra, caracterizado pela intensa industrialização, aumento populacional e do uso de recursos naturais, a sociedade passou a discutir e considerar limites para o crescimento econômico, tendo em vista a evidente finitude de recursos naturais que pudessem sustentar o modo de vida e produção da sociedade moderna de forma equilibrada.

É relevante citar, neste contexto, a criação do Clube de Roma, em 1968, no qual um grupo de líderes, personalidades e cientistas alertou sobre os riscos de um crescimento econômico contínuo, com base nos recursos naturais esgotáveis, elaborando o conhecido relatório denominado Limites do Crescimento.

A percepção do desequilíbrio instaurado e seus efeitos adversos sobre a biodiversidade levaram diversos países a elaborarem políticas públicas voltadas à regulamentação de atividades nocivas ao meio ambiente. No Brasil, o Conselho Nacional do Meio Ambiente (CONAMA) emitiu resoluções definindo o que é impacto ambiental e esta definição envolve a saúde, a segurança e o bem-estar da população, as atividades 
sociais e econômicas, a biota, as condições estéticas e sanitárias do meio ambiente e a qualidade dos recursos ambientais.

Neste sentido os impactos ambientais relacionados às atividades antrópicas podem ser avaliados em dois contextos específicos: (a) A AIA passados: são os impactos que já ocorreram, ou seja, são os passivos ambientais. Este tipo de avaliação é comum no caso de venda ou fusão de empresas, servindo ainda como subsídio ao estabelecimento de processos de recuperação de áreas degradadas e (b) A AIA futuros: trata-se de prever potenciais impactos decorrentes da instalação e/ou operação de determinada atividade ou empreendimento sobre os componentes ambientais, no sentido de estabelecer medidas que possam evitar ou minimizar a ocorrência destes impactos.

Considerando que a biodiversidade é entendida como a variabilidade de organismos vivos de todas as origens, tais como: os ecossistemas terrestres, marinhos, aquáticos e seus complexos ecológicos e a sua conservação pressupõe ações em caráter preventivo. Assim, a AIA, realizada antes que as intervenções antrópicas de fato ocorram, torna-se essencial para a conservação da biodiversidade em todos os seus níveis e aspectos. Porém, inúmeras críticas vêm sendo feitas em relação à efetividade do processo de AIA entre eles a não incorporação da componente biodiversidade ou a insuficiência na abordagem deste componente no contexto do processo.

Neste sentido, o presente trabalho abordará a análise da componente biodiversidade na realização da AIA, no contexto do licenciamento ambiental de empreendimentos potencialmente causadores de impactos ambientais, enfatizando aspectos específicos que devem ser considerados para que a capacidade dos sistemas naturais e processos ecológicos sejam protegidos durante os processos de tomada de decisão.

\section{BREVE HISTÓRICO DA AIA NO BRASIL}

A AIA é um instrumento mundialmente utilizado para analisar a viabilidade ambiental de empreendimentos/atividades (MORGAN, 2012). No Brasil, as primeiras experiências com AIA ocorreram antes mesmo da promulgação da Lei n ${ }^{\circ}$ 6.938/1981 conhecida como Política Nacional de Meio Ambiente (PNMA). Por exigência do Banco Mundial, influenciado pelas reuniões técnicas preparatória da Organização das Nações Unidas (ONU) para a Conferência das Nações Unidas para o Meio Ambiente, que foi realizada em 1972, em Estocolmo. No Brasil, a primeira norma relacionada a AIA foi a Resolução CONAMA nº 001, de 23 de janeiro de 1986, definiu impacto ambiental como

Art. $1^{\circ}[\ldots]$ qualquer alteração das propriedades físicas, químicas e biológicas do meio ambiente, causada por qualquer forma de matéria ou energia resultante das atividades humanas que, direta ou indiretamente, afetam:

I - a saúde, a segurança e o bem-estar da população;

II - as atividades sociais e econômicas;

III - a biota;

IV - as condições estéticas e sanitárias do meio ambiente;

V - a qualidade dos recursos ambientais (BRASIL, Resolução/CONAMA 001/1986, Art. 1º, Inciso I, II, III, IV e V)

O empreendimento dessa análise foi o projeto de instalação da Hidrelétrica de Sobradinho, que deveria apresentar uma AIA neste mesmo ano (1972), como condição de aprovação de empréstimos e financiamentos. O estudo em questão, porém, se concentrou nos impactos que o meio ambiente poderia ocasionar na operação do empreendimento, e não o contrário. Apenas em 1981 a AIA foi institucionalizada, em nível federal, pela Lei no 6938/1981. Esta norma legal apresenta a AIA como um dos instrumentos para a aplicação da PNMA, em seu Artigo $9^{\circ}$, inciso III.

O Decreto $n^{\circ} 88.351 / 1983$, substituído pelo Decreto $n^{\circ} 99.274 / 1990$, regulamentou a Lei $n^{\circ}$ 6.938/1981 e vinculou a AIA aos sistemas de licenciamento, outorgando ao CONAMA a competência para 
fixar os critérios básicos segundo os quais serão exigidos os Estudos de Impacto Ambiental (EIA) para fins de licenciamento, em seu artigo 17 , parágrafo $1^{\circ}$ o decreto afirma que

Art. 17. A construção, instalação, ampliação e funcionamento de estabelecimento de atividades utilizadoras de recursos ambientais, consideradas efetiva ou potencialmente poluidoras, bem como os empreendimentos capazes, sob qualquer forma, de causar degradação ambiental, dependerão de prévio licenciamento do órgão estadual competente, integrante do SISNAMA, sem prejuízo de outras licenças legalmente exigíveis.

$\int 1^{\circ}$ Caberá ao CONAMA fixar os critérios básicos, segundo os quais serão exigidos estudos de impacto ambiental para fins de licenciamento, contendo, entre outras, os seguintes itens: a) diagnóstico ambiental da área; b) descrição da ação proposta e suas alternativas; c) identificação, análise e previsão dos impactos significativos, positivos e negativos (BRASIL, Decreto no 99.274/1990, Art. 17 Caput e $\int 1^{\circ}$ ).

É importante destacar que a PNMA e sua regulamentação foram recepcionadas pela Constituição da República Federativa do Brasil (CRFB), de 1988, o Artigo 225 desta constituição prevê que "Todos têm direito ao meio ambiente ecologicamente equilibrado, bem de uso comum do povo e essencial à sadia qualidade de vida, impondo-se ao Poder Público e à coletividade o dever de defendê-lo e preservá-lo para as presentes e futuras gerações" (CRFB, 1988, Art. 225).

$\mathrm{O} \int 1^{\circ}$ deste artigo destaca que, para assegurar a efetividade desse direito, incumbe ao Poder Público, entre outros, exigir, na forma da lei, para instalação de obra ou atividade potencialmente causadora de significativa degradação do meio ambiente, estudo prévio de impacto ambiental, a que se dará publicidade.

Em 1986, por meio da Resolução CONAMA nº 001 foram estabelecidos os critérios básicos para os EIA e respectivo Relatório de Impacto Ambiental (RIMA), contendo a AIA e a lista dos empreendimentos considerados de significativo impacto ambiental e, portanto, sujeitos ao EIA e ao RIMA. A Resolução CONAMA n ${ }^{\circ} 237$ de 1997 foi criada com o objetivo de revisar os procedimentos e regular os aspectos, de forma a propiciar uma efetiva utilização do instrumento do licenciamento ambiental como forma para uma gestão ambiental otimizada, ampliando também o rol das atividades sujeitas aos EIA.

\section{A AIA NOS PROCESSOS DE LICENCIAMENTO AMBIENTAL}

O licenciamento ambiental, assim como a AIA, é um dos instrumentos da PNMA de 1981. O Art. $1^{\circ}$, Inciso I, da Resolução CONAMA nº 237 de 1997 define Licenciamento Ambiental como

[...] procedimento administrativo pelo qual o órgão ambiental competente licencia a localização, instalação, ampliação e a operação de empreendimentos e atividades utilizadoras de recursos ambientais, consideradas efetiva ou potencialmente poluidoras ou daquelas que, sob qualquer forma, possam causar degradação ambiental, considerando as disposições legais e regulamentares e as normas técnicas aplicáveis ao caso (BRASIL, Resolução/CONAMA 237/1997, Art. $1^{\circ}$, Inciso I).

O procedimento administrativo que subsidia a concessão da licença ambiental é composto por um complexo de etapas necessárias à avaliação do cumprimento dos padrões ambientais exigíveis, desde a concepção da atividade ou empreendimento até a sua operação e, durante todo tempo de vida útil do mesmo. Assim, as etapas do licenciamento ambiental são definidas pela concessão das seguintes licenças, de acordo com a Resolução CONAMA nº 237/1997:

I - Licença Prévia (LP) - concedida na fase preliminar do planejamento do empreendimento ou atividade aprovando sua localização e concepção, atestando a viabilidade ambiental e estabelecendo os requisitos básicos e condicionantes a serem atendidos nas próximas fases de sua implementação;

II - Licença de Instalação (LI) - autoriza a instalação do empreendimento ou atividade de acordo com as especificações constantes dos planos, programas e projetos aprovados, incluindo as medidas de controle 
ambiental e demais condicionantes, da qual constituem motivo determinante;

III - Licença de Operação (LO) - autoriza a operação da atividade ou empreendimento, após a verificação do efetivo cumprimento do que consta das licenças anteriores, com as medidas de controle ambiental e condicionantes determinados para a operação (BRASIL, Resolução/CONAMA 237/1997, Art. 8, Incisos I, II e III).

A AIA é parte imprescindível do EIA, sendo este apresentado como subsídio à concessão da licença prévia. De forma geral, o EIA apresenta uma estrutura básica, com uma itemização mínima necessária (CONAMA 001/1986; CONAMA 237/1997; SANCHEZ, 2006), apresentada a seguir:

a) Introdução e justificativa: apresenta os fatos que comprovam a demanda social existente pelo empreendimento/atividade.

b) Estudo de alternativas locacionais: consiste na análise ambiental integrada de todo o território com o objetivo de buscar o local com maior aptidão para a implantação do empreendimento/atividade pretendido, ou seja, o local com menor potencial de deflagração de impactos ambientais.

c) Descrição do projeto básico de engenharia: apresentação dos detalhes gerais do empreendimento, como área construída, tipo de materiais, equipamento de controle ambiental, entre outros.

d) Diagnóstico ambiental: envolve a caracterização dos meios físico, biótico em antrópico com diferentes escalas de análise, definidas pela delimitação das áreas de influência do empreendimento.

e) Avaliação de Impactos Ambientais: consiste em identificar os possíveis impactos futuros e avalia-los conforme critérios que definem sua significância, de acordo com metodologias específicas.

f) Medidas mitigadoras e/ou compensatórias: detalhamento dos controles ambientais a serem adotados para minimizar ou até evitar determinados impactos, bem como medidas de compensação ambiental para os impactos não mitigáveis.

g) Planos de acompanhamento e monitoramento: conjunto de procedimentos para a avaliação da eficácia dos controles ambientais a serem adotados.

h) Conclusão: trata do posicionamento final da equipe multidisciplinar do EIA a respeito da viabilidade ambiental do empreendimento, frente aos compromissos assumidos pelo empreendedor, explicitados no estudo.

É importante destacar que esta estrutura é variável, já que os termos de referência expedidos pelos órgãos ambientais abrangem especificidades regionais, políticas e operacionais. Porém, pode-se verificar que a AIA é o conector de todas as etapas do licenciamento, já que o empreendedor deve implantar as medidas mitigadoras e compensatórias dos impactos apontados pela AIA e avaliar a sua eficiência desde a implantação do empreendimento até o fim da vida útil deste. Isso acontece por meio dos Planos de Controle Ambiental (PCA) e respectivo Relatório de Controle Ambiental (RCA), solicitados pelos órgãos ambientais, geralmente em ocasião da emissão da Licença de operação e de sua renovação periódica.

\section{A AIA NO CONTEXTO DA BIODIVERSIDADE}

A Convenção sobre a Diversidade Biológica (CBD), reconhece a AIA como um importante instrumento de apoio à decisão ao ajudar a planejar e a implementar o desenvolvimento com foco na biodiversidade. Áreas considerada como de biodiversidade importante são aquelas que, de acordo com Associação Internacional para Avaliação de Impacto (IAIA, 2005):

a) Suportam habitats, espécies ou genótipos endémicos, raros ou em declínio.

b) Suportem genótipos ou espécies cuja presença é um pré-requisito à persistência de outras espécies.

c) Funcionam como um tampão, um habitat de ligação ou corredor ecológico ou desempenham um papel importante na manutenção da qualidade do ambiente.

d) Têm uma importante utilização sazonal ou são críticas para a migração.

e) Suportam habitats, populações ou ecossistemas vulneráveis, ameaçados ao longo da sua distribuição e cuja recuperação é lenta. 
f) Suportam áreas particularmente extensas ou continuadas de habitats anteriormente não perturbados.

g) Funcionam como um refúgio da biodiversidade durante as mudanças climáticas, permitindo a persistência e continuidade dos processos evolutivos.

h) Suportam biodiversidade para a qual a mitigação de impactos é difícil ou cuja eficácia não está comprovada, incluindo habitats que levam muito tempo a desenvolver biodiversidade característica.

i) Se apresentam atualmente pobres em biodiversidade, mas têm o potencial para desenvolver elevada biodiversidade, se for realizada uma intervenção apropriada.

Grandes empreendimentos de infraestrutura, tais como usinas hidrelétricas, rodovias, portos, linhas de transmissão, mineração, atividades petrolíferas, entre outros, normalmente ocupam grandes áreas que, inevitavelmente, comportam ecossistemas com características similares às elencadas anteriormente.

A IAIA estabelece alguns princípios orientadores e operacionais para a AIA no contexto da conservação da biodiversidade. Os principais princípios orientadores destacados pela IAIA são:

a) Visar a Conservação e a "Paragem de Perda Líquida" ("no net loss") de Biodiversidade: A biodiversidade tem que ser conservada para assegurar a continuidade no fornecimento de serviços, valores e benefícios para as gerações atuais e futuras, dentro da seguinte abordagem:

- Evitar perdas irreversíveis de biodiversidade.

- Procurar soluções alternativas que minimizem as perdas de biodiversidade.

- Utilizar a mitigação para restaurar os recursos da biodiversidade.

- Compensar as perdas inevitáveis fornecendo substitutos com um valor de biodiversidade, pelo menos, semelhante.

- Procurar oportunidades de melhoria.

b) Adotar uma abordagem ecossistêmica: a sociedade e a biodiversidade dependem de ecossistemas que funcionem de forma equilibrada e sustentável. Sendo assim, devem ser avaliados de forma integrada, sem fronteiras artificiais.

c) Procurar o uso sustentável dos recursos da biodiversidade. Utilizar a AIA para identificar, proteger e promover o uso sustentável da biodiversidade, de modo a que os resultados sejam mantidos ao longo do tempo.

d) Garantir a repartição equitativa: buscar alternativas que não troquem o “capital” de biodiversidade pela satisfação de necessidades de curto prazo, nos casos em que isto possa comprometer a capacidade das gerações futuras de satisfazerem as suas necessidades.

e) Aplicar o Princípio da Precaução: utilizar o princípio sempre que biodiversidade importante possa estar ameaçada e o conhecimento seja insuficiente para quantificar os riscos ou para implementar medidas de mitigação efetivas.

f) Adotar uma abordagem participativa: Efetuar uma consulta alargada para garantir que todas as partes interessadas foram consultadas e que os valores importantes da biodiversidade foram considerados.

Com relação aos princípios operacionais, são destacados os principais:

a) Seleção de ações: devem ser utilizados critérios de seleção para a AIA, incluindo minimamente:

- Impactos potenciais sobre áreas protegidas ou áreas que suportem espécies protegidas.

- Impactos sobre outras áreas, não protegidas, mas importantes para a biodiversidade

- Atividades que apresentem ameaças específicas para a biodiversidade (em termos do seu tipo, dimensão, localização, duração, reversibilidade).

- Áreas que proporcionem importantes serviços da biodiversidade, incluindo reservas extrativistas, territórios de populações indígenas, zonas húmidas, áreas de reprodução de peixes, solos propensos à erosão, habitats característicos ou relativamente intactos, leitos de cheias, áreas de recarga de aquíferos, etc.

b) Definição de âmbito: a definição de âmbito conduz aos Termos de Referência da AIA, definindo as questões a serem estudadas e os métodos a serem usados.

c) Estudo de impacto e preparação do relatório: abordar a biodiversidade a todos os níveis apropriados 
e conceder tempo suficiente para considerar os efeitos sazonais nos levantamentos.

d) Mitigação: as ações corretivas podem assumir perspectivas de prevenção, mitigação ou compensação. Devem-se buscar alternativas para potencializar positivamente a biodiversidade e considerar que a compensação nem sempre será possível. Poderão existir casos nos quais é conveniente dizer "não" às propostas de desenvolvimento, perante situações de dano irreversível para a biodiversidade.

e) Tomada de decisão: considerar a aplicação do princípio da precaução quando a informação for insuficiente

f) Gestão, monitorização, avaliação e auditoria: as previsões em relação à resposta da biodiversidade aos fatores de perturbação são incertas, especialmente em cenários de longo prazo. Assim, devem ser realizadas auditorias regulares dos impactos na biodiversidade. Deverão ser previstas medidas de resposta a emergências e/ou planos de contingência, sempre que circunstâncias perturbadoras ou acidentais possam ameaçar a biodiversidade.

\section{DESAFIOS ATUAIS E FUTUROS}

Apesar de observado um considerável progresso na aplicação da AIA no contexto das questões da biodiversidade, existe ainda muitos desafios a serem superados. Trajano (2010) apresenta importantes críticas no sentido de que os impactos cumulativos e sinérgicos de empreendimentos, até daqueles não sujeitos legalmente à AIA, não estão sendo considerados, mesmo que a multitude de intervenções individualmente menores represente ameaça relevante à biodiversidade. Também destaca que, apesar de reconhecidas as Áreas Prioritárias para a Conservação, Utilização Sustentável e Repartição dos Benefícios da Biodiversidade Brasileira, por meio da Portaria no 126, de 2004 do Ministério do Meio Ambiente, estas não vêm sendo consideradas nos processos de licenciamento do uso de recursos naturais, comprometendo os objetivos estabelecido no Artigo $1^{\circ}$ da referida portaria, que são:

I - Conservação in situ da biodiversidade;

II - Utilização sustentável de componentes da biodiversidade;

III - Repartição de benefícios derivados do acesso a recursos genéticos e ao conhecimento tradicional associado;

IV - Pesquisa e inventários sobre a biodiversidade;

V - Recuperação de áreas degradadas e de espécies sobre-exploradas ou ameaçadas de extinção; e

VI - Valorização econômica da biodiversidade. (BRASIL, MMA, Portaria no 126/2004, art. 1º, incisos I, II, III, IV, V, VI).

Constata-se que os valores da biodiversidade são frequentemente subestimados nos processos de licenciamento de empreendimentos de significativos impactos ambientais realizados atualmente no Brasil. Não costumam ser considerados os valores econômicos da biodiversidade, os valores sociais e outros valores intrínsecos. Um exemplo bastante conhecido é o caso da Usina Hidrelétrica de Belo Monte. A obra, situada no Rio Xingu, no Estado do Pará, e é alvo de amplos debates e discussões relativos aos impactos ambientais decorrentes, além dos sociais. Em termos de biodiversidade, de acordo com Medeiros (2009), existem erros claros na identificação de espécies da fauna aquática do rio Xingu, que apresenta uma das maiores riquezas de espécies do planeta.

Além disso, Fearneside (2009) indicou diversas outras omissões e falhas no EIA, que impedem análises conclusivas sobre temas considerados chave. Constatações similares foram feitas em relação ao EIA que subsidiou a emissão da Licença Prévia das Obras de transposição do Rio São Francisco, em 2005 (com uma posterior Licença Prévia Retificatória em 2006), defendida desde o século XIX como a solução para os problemas do Nordeste. Além de inúmeras omissões, como, por exemplo, extinção de espécies da fauna e da flora, verificou-se que o EIA não apresentou uma análise global e integrada das consequências da execução do projeto. 
Para Carmo (2016), apesar do importante papel da AIA na mitigação de impactos e na melhoria de muitos projetos apresentados, não parece adequada à realidade de um número elevado de grandes empreendimentos. Turra et al., (2017) apontam que a visão fragmentada e reducionista do licenciamento de projetos inicia-se pela etapa do escopo dos EIA, materializado pelo Termo de Referência (TR) no Brasil. Neste contexto, as questões relativas à biodiversidade merecem especial atenção, já que são tratadas, muito frequentemente, de forma insípida ou mesmo desconsideradas ao longo do processo.

Septanil (2017) analisou a inserção de informações sobre a biodiversidade na etapa de escopo da AIA no Brasil (2005-2014) e constatou que tais informações não foram inseridas de maneira satisfatória nos termos de referência. Francisco et al., (2014) analisando o conteúdo e o nível de detalhamento trazido pelos EIA elaborados para empreendimentos rodoviários do Estado de São Paulo no que tange à temática da biodiversidade, identificaram omissões importantes para a caracterização completa da biodiversidade em seus três níveis. Os EIA apresentaram predomínio de dados secundários para os níveis de espécies e ecossistemas, também apresentando ausência de qualquer informação sobre diversidade genética. A AIA não trata da perda de variabilidade genética, aumento do risco de extinção ou invasão biológica.

Almeida (2010), analisando os diagnósticos de meio biótico em estudos ambientais aprovados por órgão ambientais em Minas Gerais, identificou baixa qualidade de dados apresentados. Scherer (2011) realizou uma análise crítica dos aspectos referentes à fauna de cinco EIA realizados no contexto do Bioma Mata Atlântica. Os resultados identificaram carências, tais como: (a) ausência de informação sobre invertebrados terrestres; (b) ausência da identificação de áreas de reprodução e alimentação; (c) identificação superficial de fenômenos de migração e de aspectos comportamentais; (d) caracterização superficial das interações ecológicas; (e) ausência da identificação da estrutura das comunidades presentes e espécies-chave; e (f) ausência da identificação de bioindicadores. A maioria dos EIA não realizou um diagnóstico do meio biótico suficientemente adequado para subsidiar a posterior avaliação de impactos.

Para abarcar minimamente as questões relevantes em termos de biodiversidade, a IAIA recomenda que a AIA contemple diferentes níveis de análise para avaliar a viabilidade ambiental de uma atividade/empreendimento, conforme apresenta a Quadro 1.

Quadro 1 - Níveis a serem considerados na AIA para avaliar a viabilidade ambiental de atividade/empreendimento em relação a componente biodiversidade

\begin{tabular}{|c|c|c|}
\hline NÍVEL & & QUESTIONAMENTO \\
\hline \multirow{4}{*}{ Genético } & \multirow{4}{*}{$\begin{array}{l}\text { Até que ponto a } \\
\text { proposta terá efei- } \\
\text { tos significativos } \\
\text { sobre: }\end{array}$} & $\begin{array}{l}\text { A diversidade genética das espécies, particularmente as espécies raras e em declínio } \\
\text { e as identificadas como prioritárias em Estratégias Nacionais e Planos de Ação para } \\
\text { a Biodiversidade? }\end{array}$ \\
\hline & & $\begin{array}{l}\text { As oportunidades de interação entre as populações das espécies, por exemplo, ao } \\
\text { aumentar a fragmentação do habitat e o isolamento? }\end{array}$ \\
\hline & & O risco de extinção? \\
\hline & & A persistência de espécies localmente bem adaptadas? \\
\hline \multirow{5}{*}{ Específico } & \multirow{5}{*}{$\begin{array}{l}\text { Até que ponto a } \\
\text { proposta irá: }\end{array}$} & $\begin{array}{l}\text { Alterar a riqueza das espécies ou a composição específica dos habitats na área de } \\
\text { estudo? }\end{array}$ \\
\hline & & Alterar a composição específica das comunidades? \\
\hline & & Provocar o desaparecimento de algumas espécies da área? \\
\hline & & $\begin{array}{l}\text { Afetar espécies identificadas como prioritárias nas Estratégias Nacionais e Planos de } \\
\text { Ação para a Biodiversidade? }\end{array}$ \\
\hline & & Aumentar o risco de invasão por espécies exóticas? \\
\hline \multirow{3}{*}{ Ecossistêmico } & \multirow{3}{*}{$\begin{array}{l}\text { Até que ponto a } \\
\text { proposta irá: }\end{array}$} & Alterar a quantidade, qualidade ou organização espacial do habitat? \\
\hline & & Afetar planos para melhorar a disponibilidade ou qualidade do habitat? \\
\hline & & $\begin{array}{l}\text { Provocar danos nos processos e serviços do ecossistema, particularmente aqueles } \\
\text { dos quais dependem as comunidades locais? }\end{array}$ \\
\hline
\end{tabular}

Fonte: IAIA, 2005.

A verificação da efetividade dos diagnósticos ambientais, no sentido de identificar a inserção da biodiversidade, em termos legais e científicos, é de suma importância na avaliação dos EIA, visando garantir 
sua conservação em um padrão aceitável.

\section{CONSIDERAÇÕES FINAIS}

A AIA assume importância central no processo de licenciamento ambiental de empreendimentos de significativo impacto ambiental, assim definidos pelas Resoluções do CONAMA n 001/86 e n² 237/87. Porém, a aplicação deste importante instrumento da PNMA vem se mostrando útil, muitas vezes, apenas parte de um processo administrativo, já que as práticas se distanciam do ideal normativo.

Investigações rasas, muitas vezes até ausentes, acerca de especificidades ecossistêmicas, diversidade genética, interações ecológicas, identificação da estrutura das comunidades presentes e espécies-chave, bioindicadores, entre outros, podem conduzir a tomadas de decisão equivocadas em relação à real viabilidade ambiental de inúmeros empreendimentos. Os EIA e estudos correlatos subsidiam a emissão das licenças ambientais que, quando concedidas, passam à sociedade a ideia de que o empreendimento/atividade está em sintonia com o desenvolvimento sustentável.

A legislação ambiental brasileira é complexa e abrangente, mas carece de esforços em sua estrutura institucional, visto que grande parte dos licenciamentos ocorre no âmbito estadual. Estes órgãos ambientais, muitas vezes, não apresentam um nível de exigência de informações adequado para a análise de viabilidade de empreendimentos/atividade potencialmente causadores de significativo impacto ambiental, contribuindo assim para a perda sistemática de biodiversidade.

Para que a AIA, como instrumento da PNMA, cumpra seus objetivos é fundamental a inserção da componente biodiversidade no escopo dos EIA, em nível de levantamento primário de informações, em escalas espaciais e temporais adequadas e em grau de detalhamento e aprofundamento coerentes com as questões a serem compreendidas.

\section{REFERÊNCIAS}

ALMEIDA, M. R. R. Análise da qualidade de Relatórios de Controle Ambiental aprovados pela Superintendência Regional de Meio Ambiente e Desenvolvimento Sustentável do Sul de Minas Gerais. Dissertação de Mestrado. Universidade Federal de Itajubá, 154 p, 2010.

BRASIL. Lei No 6.938, de 31 de agosto de 1981 - Dispõe sobre a Política Nacional do Meio Ambiente, seus fins e mecanismos de formulação e aplicação, e dá outras providências. Brasília: Diário Oficial da União de 02 de setembro de 1981.

Constituição da República Federativa do Brasil, de 1988. Brasília: Senado Federal, SecretariaGeral da Mesa, 2001.

. Decreto $n^{\circ} .99 .274 / 90$ de 6 de junho de 1990. Regulamenta a Lei $\mathbf{n}^{\circ} \mathbf{6 . 9 3 8}$, de 31 de agosto de 1981, sobre a Política Nacional do Meio Ambiente, e dá outras providências. Diário Oficial da União: Brasília, edição de 7 de junho de 1990.

. Conselho Nacional do Meio Ambiente - CONAMA. Resolução CONAMA 01, de 23 de janeiro de 1986. Dispõe sobre procedimentos relativos a Estudo de Impacto Ambiental. Publicada no Diário Oficial da União, de 17/02/1986, p. 2548-2549.

Conselho Nacional do Meio Ambiente CONAMA. Resolução CONAMA 237 de 19 de dezembro de 1997. Publicada no Diário Oficial da União em 22.12.1997; com retificação em 13.10.2003.

. MMA (Ministério do Meio Ambiente). Portaria no 126, de 27 de maio de 2004 Área Prioritárias para Conservação, Uso Sustentável e Repartição de Benefícios da Biodiversidade brasileira: atualização - Portaria MMA no 09, de 23 de janeiro de 2007. Secretaria de Biodiversidade e Florestas, MMA, Brasília. Disponível em:http://www.mma.gov.br/estruturas/chm/_arquivos/biodiversidade31.pdf. Acesso em 3 abr. 2019.

CARMO, A. B. Avaliação de Impacto Ambiental em empreendimentos costeiros e marinhos no 
Brasil: análise dos procedimentos e aspectos institucionais e políticos. Tese (Doutorado em Oceanografia) - Instituto Oceanográfico, Universidade de São Paulo, São Paulo, 2016.

FEARNESIDE, P. M. O Novo EIA-RIMA da Hidrelétrica de Belo Monte: Justificativas Goela Abaixo. Em: Painel de Especialistas - Análise Crítica do Estudo de Impacto Ambiental do Aproveitamento Hidrelétrico de Belo Monte. International Rivers, 2009.

FRANCISCO, R. T. et al. Avaliação de impactos ambientais e conservação da biodiversidade em empreendimentos de infraestrutura rodoviária do estado de São Paulo. In: SIICUSP - $22^{\circ}$ Simpósio Internacional de Iniciação Científica e Tecnológica da USP. 4p, 2014.

IAIA, International Association for Impact Assessment. Biodiversity in Impact Assessment. Fargo: IAIA, Special Publication Series No. 3, 2005.

MEDEIROS, Hermes Fonseca. Avaliação de Impactos do Projeto de Aproveitamento Hidrelétrico de Belo Monte Sobre a Vida Selvagem, Incluindo Implicações Socioeconômicas. In: Painel de Especialistas: Análise Crítica do Estudo de Impacto Ambiental do Aproveitamento Hidrelétrico de Belo Monte. 2009.

MORGAN, R. K. Environmental impact assessment: the state of the art. Impact Assessment and Project Appraisal, v. 1, n. 30, p. 5-14, 2012.

SANCHEZ, Luis Henrique. Avaliação de impacto ambiental: conceitos e métodos. São Paulo: Oficina de Textos, 2006.

SCHERER, M. Análise da qualidade técnica de estudos de impacto ambiental em ambientes de Mata Atlântica de Santa Catarina: abordagem faunística. Revista Biotemas, v 24 n 4, p 171-181, 2011.

SEPTANIL, Moema Pauline Barão. A inclusão da biodiversidade na Avaliação de Impacto Ambiental: análise da etapa de escopo no estado de São Paulo. 2017. 225 p. Dissertação (Mestrado em Ciências da Engenharia Ambiental) - Escola de Engenharia de São Carlos, Universidade de São Paulo, São Carlos, 2017. TRAJANO, E. Políticas de conservação e critérios ambientais: princípios, conceitos e protocolos. Estudos Avançados, v 24, n 68, p 135-146, 2010.

TURRA, A. et al. Avaliação de impacto ambiental sob uma abordagem ecossistêmica: ampliação do porto de São Sebastião. Ambiente \& Sociedade. v. XX, n. 3, p. 159-178, jul.-set. 2017. 